BULLETIN OF THE

AMERICAN MATHEMATICAL SOCIETY

Volume 78, Number 4, July 1972

\title{
SET VALUED TRANSFORMATIONS
}

\author{
BY D. G. BOURGIN
}

Communicated by Morton Curtis, December 2, 1971

The present note summarizes some results in a new algebraic topological approach to set valued transformations and initiates a theory of their fixed points applicable to the case that the images are not acyclic. These ideas extend to min max theorems where again a basic generalization is obtained [2]. Our developments are based on the existence of homomorphisms of certain homology groups, in a crucial range only, induced by suitably defined multivalued homotopies (cf. Theorems 1 and 3 below).

Let $X$ and $Y$ be paracompact spaces and suppose $h: X \times I \rightarrow Y$ is a set valued uppersemicontinuous (usc) transformation. Let $\Gamma(h)$ be the graph

$$
\Gamma(h)=\bigcup\{(x, s, y) \mid y \in h(x, s)\} \subset X \times I \times Y .
$$

Let $p_{1}$ be the projection of $\Gamma(h)$ onto $X, p_{2}$ the projection onto $Y$ and $P_{1}$ the projection of $\Gamma(h)$ onto $X \times I$.

For each $s \in I$ the singular set $S^{p}(s)$ is defined by

$$
S^{p}(s)=\left\{x \mid H^{r} h(x, s) \neq 0 \text { for some } r<p\right\}
$$

where $H^{*}$ refers to Alexander reduced cohomology over the coefficient field $Q$ and closed support family. Write

$$
S^{p}=\bigcup_{s \in I} S^{p}(s)
$$

We say $p_{1}$ is almost $p$ solid, ApS, if for any neighborhood $N\left(y_{0}\right)$ in a suitable neighborhood base at $y_{0} \in Y$, there is at most a finite subset of $S^{p}$, independent of $s$, such that $h(x, s) \cap N\left(y_{0}\right) \neq \varnothing, x \in S^{p}$, does not imply $h(x, s) \in N\left(\gamma_{0}\right)$ and $h(x, s)$ is uniformly usc for fixed $x$.

We write $f \sim_{p q} g$ if $h_{s}, s \in I$, is acyclic for $p \leqq m \leqq q \leqq \infty$ and $p_{1}(h)$ is ApS. The basic theorem for our purpose is

THEOREM 1. If $f \sim_{p q} g, q \geqq p+2$ and $h$ describes the homotopy then $h(m)^{*}: H^{m}(Y) \rightarrow H^{m}(X \times I)$ exists for $p+2 \leqq m \leqq q$ and $f^{*}(m)=g^{*}(m)$ for this range of $m$ values.

If $X$ and $Y$ are compacta, a condition designated by (C) is

AMS 1970 subject classifications. Primary 54C60, 54H25, 49A40; Secondary 55C20.

Key words and phrases. Set valued transformation, uppersemicontinuous, paracompact, graph, homotopy, almost $p$ solid, Alexander cohomology, min max. 
$S^{p}$ is denumerable and for arbitrary positive $\varepsilon$, there is at most a finite subset of $S^{p}$ for which diam $f(x)>\varepsilon$. As a consequence of Theorem 1 restricted to compact spaces there results

THEOREM 2. Let $f$ be an uppersemicontinuous set valued self transformation of the $n+1$ disk, $D^{n+1}, n>3$. Let $S^{n-2}$ be the singular subset defined by the condition that $f(x)$ is a convex set for $x \bar{\epsilon} S^{n-2}$ and for $x \in S^{n-2}$, $f(x)$ is a finite union of convex sets of which at most $n-1$ are of dimension greater than $n-3$. Require also that (C) be satisfied. Then $f$ has a fixed point.

Another type of homotopy theorem is also available. Thus for a set valued transformation $f$ on $X$ to $Y$ we grade the singular set by

$$
\mu_{r}=\left\{x \mid H^{r} f(x) \approx 0\right\} .
$$

Let $d_{r}=\operatorname{dim} \mu_{r}$ the maximum covering dimension of sets $A$ closed in $Y$ and contained in $\mu_{r}$. Similarly for $h$,

$$
V_{r}=\left\{(x, s) \mid H^{r} h(x, s) \approx 0\right\} .
$$

Let $\delta_{r}=\operatorname{dim} V_{r}$ be the maximum covering dimension of sets $A$ closed in $X \times I$ and contained in $V_{r}$. Let

$$
\Pi=1+\sup _{V_{r} \neq \varnothing ; r<q}\left(r+\delta_{r}\right) .
$$

The notation $f \sim_{p \Pi_{q}} g$ is used if there is a usc transformation $h$, said to describe the homotopy with $V_{r}=\varnothing, p \leqq r \leqq q$ and $p \leqq \Pi<q$.

The correspondent to Theorem 1 is

THEOREM 3. If $f \sim_{p \Pi q} g$ and $h$ describes this homotopy with $f, g$ and $h$ usc then if $q \geqq \Pi+2, h^{*}(m)$ exists and $f^{*}(m)=g^{*}(m)$ for $\Pi+1 \leqq m$ $<q$.

For the case that $\delta_{r} \equiv 0$ for all $r<p$, Theorem 3 includes Theorem 1 if the spaces are compacta. (However this is not true if either the compactness or the metrizability restrictions are dropped as can be shown by suitable examples.) Accordingly instead of Theorem 2 we can assert

THEOREM 4. Let $f$ be a usc transformation on $D^{n+1}$ to $D^{n+1}$. Let $S$ be the singular set $\bigcup V_{r}$ with $\operatorname{dim} S=d$. Suppose $\mu_{r}=\varnothing$ for $r \geqq n-3-d=\bar{r}$. For $x \bar{\in} S, f(x)$ is convex. For $x \in S, f(x)$ is the finite union of convex sets with at most $\bar{r}+1$ of dimension greater than $\bar{r}-1$. Then f has a fixed point.

The results above have immediate application to the central theorem of game theory, namely, the min max theorem. Let $X$ and $Y$ be convex bodies in $R^{k}$ and $R^{l}$ and let $f$ be a real valued (continuous) map on $X \times Y$. A saddle point or min max point $x_{0}, y_{0}$ is defined by 


$$
\underset{y \in Y}{\operatorname{Min}} f\left(x^{0}, y\right)=f\left(x^{0}, y^{0}\right)=\underset{x \in X}{\operatorname{Max}} f\left(x, y^{0}\right) .
$$

Define

$$
\begin{aligned}
& M(y)=\left\{x \mid f(x, y)=\operatorname{Max}_{x \in X} f(x, y)\right\} \subset X, \\
& N(x)=\left\{y \mid f(x, y)=\operatorname{Min}_{y \in Y} f(x, y)\right\} \subset Y .
\end{aligned}
$$

Let $g(x, y)$ be the set valued transformation on $X \times Y$ to $X \times Y$ defined by

$$
g(x, y)=M(y) \times N(x) .
$$

Our new type of saddle point theorem is

THEOREM 5. Suppose $M$ and $N$ are usc with singular sets

$$
S(X)=\bigcup_{r} \mu_{r}(X), \quad S(Y)=\bigcup_{r} \mu_{r}(Y)
$$

Write $d(X)=\operatorname{dim} S(X), d(Y)=\operatorname{dim} S(Y)$. Suppose $\mu_{r}(X)=\varnothing$ for $r \geqq p$ and that $d X \leqq k-p-3$ and suppose too that $\mu_{r}(Y)=\varnothing$ for $r \geqq q$ and that $d Y \leqq l-q-3$. For $x \bar{\epsilon} S(X), N(X)$ is convex and for $x \in S(X), N(x)$ is a finite union of convex sets at most $p$ of which are of dimension at least $p-1$. For $y \in \bar{\epsilon}(Y), M(y)$ is convex and for $y \in S(Y), M(y)$ is a finite union of convex sets at most $q$ of which are of dimension at least $q-1$. Then there is a saddle point.

Detailed expositions and proofs of the results above will be given in [1] and [2].

\section{BIBLIOGRAPHY}

1. D. G. Bourgin, Cones and Vietoris-Begle type theorems, Trans. Amer. Math. Soc. (to appear).

2. - Fixed point and min-max theorems, Pacific J. Math. (to appear).

Department of Mathematics, University of Houston, Houston, TeXas 77004 